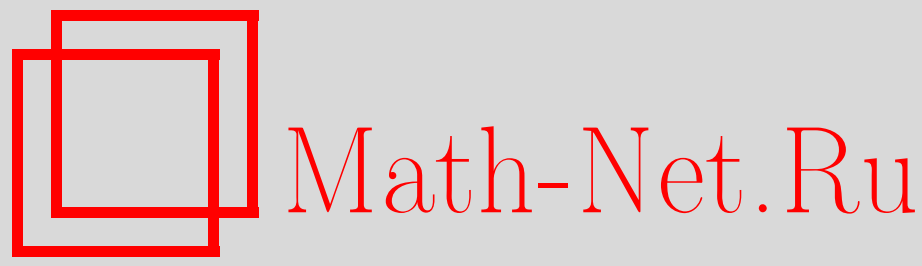

Г. Ф. Хелминк, С. В. Поленкова, Аналитический подход к двумерной бесконечной иерархии Тоды, связанной с коммутативной алгеброй, TMФ, 2008, том 155, номер 1, 177-192

DOI: https://doi.org/10.4213/tmf6202

Использование Общероссийского математического портала Math-Net.Ru подразумевает, что вы прочитали и согласны с пользовательским соглашением http: //www . mathnet.ru/rus/agreement

Параметры загрузки:

IP : 52.90 .164 .192

26 апреля 2023 г., 12:02:00

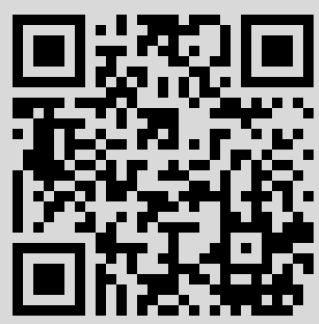




\section{АНАЛИТИЧЕСКИЙ ПОДХОД К ДВУМЕРНОЙ БЕСКОНЕЧНОЙ ИЕРАРХИИ ТОДЫ, СВЯЗАННОЙ С КОММУТАТИВНОЙ АЛГЕБРОЙ}

Исходя из группы операторов на сепарабельном гильбертовом пространстве, отличающихся от единицы на ядерный оператор, построено решение двумерной бесконечной иерархи Тоды, связанной с максимальной коммутативной подалгеброй алгебры комплексных $(k \times k)$-матриц.

Ключевые слова: двумерная бесконечная иерархия Тоды, уравнения Лакса, линеаризация, коммутирующие потоки, банахова группа Ли, большая клетка.

\section{1. ВВЕДЕНИЕ}

Иерархии тодовского типа состоят, грубо говоря, из комбинации нелинейных дифференциальных и разностных уравнений для бесконечных матриц. По поводу роли, которую иерархии Тоды играют в моделях математической физики, мы отсылаем читателя к работе [1]. Двумерная бесконечная иерархия Тоды, связанная с максимальной коммутативной подалгеброй $\mathbf{h}$ комплексных $(k \times k)$-матриц, составляющая предмет рассмотрения настоящей работы, является обобщением иерархии, рассмотренной в работе [2]. Она представляет собой комбинацию двух иерархий, одна из которых формулируется в терминах нижнетреугольных, а другая - в терминах верхнетреугольных матриц. Аналитический подход к потокам первой иерархии рассматривался в работе [3], а геометрический формализм для потоков иерархии второго типа приведен в работе [4]. В рамках формализма настоящей работы допускаются обе группы потоков и получаются решения для комбинированной иерархии. Основная цель работы состоит в доказательстве следующего результата.

Tеорема. Пусть $H=L^{2}\left(S^{1}, \mathbb{C}^{k}\right)$ - гилъбертово пространство квадратично-интегрируемых $\mathbb{C}^{k}$-значных функиий на $S^{1}, \Gamma(\mathbf{h})$ - группа обратимых голоморфных

${ }^{*}$ Korteweg de Vries Institute, University of Amsterdam, Amsterdam, The Netherlands. E-mail: helminck@science.uva.nl

${ }^{\dagger}$ Faculty of Mathematical Sciences, University of Twente, Enschede, The Netherlands. E-mail: polenkova@mail.ru 
отображений из единичной окружности $S^{1}$ в максималъную коммутативную алгебру $\mathbf{h}$ комплексных $(k \times k)$-матрии, $G_{1}$ - группа обратимых ограниченных линейных отображений пространства $H$ в себя вида "Id +ядерный оператор". Тогда справедливы следующие утверждения.

1. Для каждого $g \in G_{1}$ существует открытое подмножество $\Gamma(g, \mathbf{h})$ в $\Gamma(\mathbf{h})$ такое, что коэффициенты верхней блочно-треугольной $(k \times k)$-части $\Phi_{+}$и нижней блочно-треугольной $(k \times k)$-части $\Phi_{-}$в разложении

$$
\left[\gamma g \gamma^{-1}\right]=\Phi_{-}^{-1} \Phi_{+}, \quad \gamma \in \Gamma(g, \mathbf{h}),
$$

принадлежат кольиу $R(g, \mathbf{h})$ голоморфных функиий, заданных на открытом подмножестве $\Gamma(g, \mathbf{h})$.

2. $(\mathbb{Z} \times \mathbb{Z})$-матриць

$$
\begin{array}{clrl}
\mathcal{L}\left(\Phi_{-}\right) & :=\Phi_{-} \Lambda^{k} \Phi_{-}^{-1}, & U_{\alpha}\left(\Phi_{-}\right):=\Phi_{-} i_{k}\left(E_{\alpha}\right) \Phi_{-}^{-1}, & \alpha=1,2, \ldots, \\
\mathcal{M}\left(\Phi_{+}\right):=\Phi_{+} \Lambda^{-k} \Phi_{+}^{-1}, & V_{\beta}\left(\Phi_{+}\right):=\Phi_{+} i_{k}\left(F_{\beta}\right) \Phi_{+}^{-1}, & \beta=1,2, \ldots,
\end{array}
$$

являются решениями двумерной иерархии Тодь, связанной с $\mathbf{h}$. Здесъ $\Lambda^{-1}-$ $(\mathbb{Z} \times \mathbb{Z})$-матрица, соответствующая оператору сдвига на $H,\left\{E_{\alpha}, \alpha=1,2, \ldots\right\}$ $u\left\{F_{\beta}, \beta=1,2, \ldots\right\}$ - базисъ в $\mathbf{h}$, а $i_{k}(A)$ обозначает $(\mathbb{Z} \times \mathbb{Z})$-матрицу, соответствуюшую умножению элементов из $H$ на комплексную $(k \times k)$-матрицу $A$.

Иерархии, рассматриваемые в данной работе, сформулированы в терминах $(\mathbb{Z} \times \mathbb{Z})$-матриц. Поэтому в разделе 2 сначала введены необходимые обозначения и разложения в этом пространстве. В разделе 3 рассмотрен лаксов вид этих иерархий и соответствующая линеаризация. Заключительный раздел 4 посвящен центральному разложению в группе $G_{1}$; в этом разделе описано, как параметры непрерывных потоков в группе коммутирующих потоков $\Gamma(\mathbf{h})$ связаны с уравнениями Лакса иерархии, и это завершает доказательство теоремы.

\section{2. ПРОСТРАНСТВО $M_{\mathbb{Z}}(R)$}

Пусть $R$ - коммутативное кольцо. Введем обозначения $M_{k}(R)$ для кольца $(k \times k)$ матриц с коэффициентами в $R$ и $M_{\mathbb{Z}}(R)$ для $R$-модуля $(\mathbb{Z} \times \mathbb{Z})$-матриц с коэффициентами в $R$. Мы используем упорядочение столбцов и строк, совместное с конечномерным случаем. Имеется некоторое число специальных часто используемых элементов из $M_{\mathbb{Z}}(R)$. Прежде всего, это базисная матрица $E_{(i, j)}, i, j \in \mathbb{Z}$, задающаяся как

$$
\left(E_{(i, j)}\right)_{\mu \nu}=\delta_{i \mu} \delta_{j \nu}
$$

Любую матрицу $A=\left(A_{i j}\right) \in M_{\mathbb{Z}}(R)$ можно записать как формальную линейную комбинацию базисных матриц

$$
A=\sum_{i \in \mathbb{Z}} \sum_{j \in \mathbb{Z}} A_{i j} E_{(i, j)} .
$$

Ключевой является матрица сдвига $\Lambda=\sum_{i \in \mathbb{Z}} E_{(i-1, i)}$. В контексте данной работы она играет роль, подобную роли оператора $\partial:=d / d x$ в формализме псевдодифференциальных операторов для КП-иерархии. 
Каждому набору $\{d(s) \mid s \in \mathbb{Z}\}$ матриц из $M_{k}(R)$ можно поставить в соответствие диагональ $(k \times k)$-блоков из $M_{\mathbb{Z}}(R)$ с помощью калибровки:

$$
\operatorname{diag}(d(s)):=\sum_{s \in \mathbb{Z}} \sum_{\alpha=1}^{k} \sum_{\beta=1}^{k} d(s)_{\alpha \beta} E_{(s k+\alpha-1, s k+\beta-1)} .
$$

Пусть для каждого $k \geqslant 1$

$$
\mathcal{D}_{k}(R)=\left\{d=\operatorname{diag}(d(s)) \mid d(s) \in M_{k}(R) \quad \text { для всех } \quad s \in \mathbb{Z}\right\}
$$

обозначает кольцо блочно-диагональных $(k \times k)$-матриц из $M_{\mathbb{Z}}(R)$. Существует кольцевой гомоморфизм $i_{k}$ из $M_{k}(R)$ в $\mathcal{D}_{k}(R)$, который реализуется взятием в качестве $A \in M_{k}(R)$ всех диагональных блоков в $i_{k}(A)$, равных $A$. Элементы $\Lambda^{k m}, m \in \mathbb{Z}$, действуют на $\mathcal{D}_{k}(R)$ следующим образом:

$$
\Lambda^{k m} \operatorname{diag}(d(s)) \Lambda^{-k m}=\operatorname{diag}(d(s+m)) .
$$

Поэтому образ отображения $i_{k}$ состоит из всех матриц из $\mathcal{D}_{k}(R)$, коммутирующих с $\Lambda^{k}$. Каждую матрицу из $M_{\mathbb{Z}}(R)$ можно разбить на так называемые $(k \times k)$-блочные диагонали. Действительно, для $A=\left(A_{i j}\right) \in M_{\mathbb{Z}}(R)$ введем следующее определение.

ОПРЕДЕлЕНиЕ 2.1. Для любой матрицы $A j$-я $(k \times k)$-блочная диагональ, $j \in \mathbb{Z}$, есть матрица

$$
\sum_{i \in \mathbb{Z}} \sum_{\alpha=1}^{k} \sum_{\beta=1}^{k} A_{(k i-k j+\alpha-1, k i+\beta-1)} E_{(k i-k j+\alpha-1, k i+\beta-1)} .
$$

Из выражения $(1)$ видно, что $j$-ю $(k \times k)$-блочную диагональ $(\mathbb{Z} \times \mathbb{Z})$-матрицы $A$ можно единственным образом записать в виде $\operatorname{diag}(d(s)) \Lambda^{k j}$. Таким образом, каждую $A=\left(A_{(i, j)}\right) \in M_{\mathbb{Z}}(R)$ можно единственным образом представить как

$$
A=\sum_{j \in \mathbb{Z}} d_{j} \Lambda^{k j}
$$

где $d_{j}$ лежат в $\mathcal{D}_{k}(R)$. В частности, любая матрица, коммутирующая с $\Lambda^{k}$, имеет вид $(2)$, где $d_{j}$ лежат в образе $i_{k}$. Внутри $M_{\mathbb{Z}}(R)$ рассмотрим два подпространства, образующие кольцо по отношению к обычному произведению.

ОПРЕДЕЛЕНИЕ 2.2. Элемент $A$ из $M_{\mathbb{Z}}(R)$ называется верхним $(k \times k)$-блочно-треугольным элементом уровня $m$, если он имеет вид

$$
A=\sum_{j \geqslant m} d_{j} \Lambda^{k j}, \quad \text { где } \quad d_{j} \in \mathcal{D}_{k}(R) .
$$

Набор всех верхних $(k \times k)$-блочно-треугольных элементов уровня $m$ обозначается как $U T_{m}=U T_{m}(R)$. Мы используем обозначения

$$
U T(R):=\bigcup_{k \in \mathbb{Z}} U T_{k}=: U T
$$


для множества всех верхних $(k \times k)$-блочно-треугольных матриц. Из соотношения (1) непосредственно следует, что $U T$ является $R$-алгеброй. Противоположный класс матриц таков.

ОПРЕДЕЛЕНИЕ 2.3. Элемент $A$ из $M_{\mathbb{Z}}(R)$ называется нижним $(k \times k)$-блочно-треугольным элементом уровня $m$, если его можно записать в виде

$$
A=\sum_{j \leqslant m} d_{j} \Lambda^{k j}, \quad \text { где } \quad d_{j} \in \mathcal{D}_{k}(R) .
$$

Набор всех таких элементов обозначается как $L T_{m}=L T_{m}(R)$, и мы также используем обозначения

$$
L T(R):=\bigcup_{k \in \mathbb{Z}} L T_{k}=: L T
$$

для $R$-алгебры всех нижних $(k \times k)$-блочно-треугольных матриц.

С разложением (2) мы свяжем два обозначения: если $A=\sum_{j \in \mathbb{Z}} d_{j} \Lambda^{j}$, как в (2), то будем писать

$$
A_{+}=\sum_{j \geqslant 0} d_{j} \Lambda^{k j} \quad \text { и } \quad A_{-}=\sum_{j<0} d_{j} \Lambda^{k j}
$$

для соответствующей верхней $(k \times k)$-блочно-треугольной части и строго нижней $(k \times k)$-блочно-треугольной части $A$. Заметим, что если $U \in U T$ и $V \in L T$ имеют вид

$$
U=\sum_{i \geqslant r} u_{i} \Lambda^{i k}, \quad V=\sum_{i \leqslant r} v_{i} \Lambda^{i k},
$$

причем $u_{r}$ и $v_{r}$ обратимы в $\mathcal{D}_{k}(R)$, то элементы $U$ и $V$ обратимы, причем диагональные $(k \times k)$-блочные компоненты их обратных можно вычислить рекуррентно. Следующее утверждение показывает, что при $r \neq 0$ все обратимые элементы такого типа можно получить с помощью “одевания" матрицы $\Lambda^{k r}$.

УтВеРЖДЕНИЕ 2.1. 1. Пусть для любого ненулевого $r \in \mathbb{Z}$ элемент $\mathcal{L} \in L T_{r}$ имеет $(k \times k)$-блочный вид:

$$
\mathcal{L}=\sum_{j \leqslant r} \alpha_{j} \Lambda^{j k}, \quad \text { где } \quad \alpha_{r} \in \mathcal{D}_{k}(R) \quad \text { обратим. }
$$

Тогда существует элемент $U \in L T(k \times k)$-блочного вида, $U=\sum_{i \leqslant 0} \beta_{i} \Lambda^{k i}$, с обратимым старшим коэффициентом $\beta_{0} \in \mathcal{D}_{k}(R)$ такой, что

$$
\mathcal{L}=U \Lambda^{r k} U^{-1}
$$

Здесь элементы $\beta_{t}(i), t \leqslant 0,0 \leqslant i<|r|$, произвольны с точностъю до единственного ограничения, что элементы $\beta_{0}(i), 0 \leqslant i<|r|$, должны быть обратимы.

2. Пусть для любого ненулевого $s \in \mathbb{Z}$ элемент $\mathcal{M} \in U T_{r}$ имеет $(k \times k)$-блочный вид

$$
\mathcal{M}=\sum_{j \geqslant s} \gamma_{j} \Lambda^{k j}, \quad \text { где } \quad \gamma_{s} \in \mathcal{D}_{k}(R) \quad \text { обратим. }
$$


Тогда существует элемент $V \in U T(k \times k)$-блочного вида, $V=\sum_{i \geqslant 0} \delta_{i} \Lambda^{i k}$, с обратимой матрищей $\delta_{0} \in \mathcal{D}_{k}(R)$, удовлетворяющий равенству

$$
\mathcal{M}=V \Lambda^{s k} V^{-1} .
$$

Как и выше, элементы $\delta_{l}(j), l \geqslant 0,0 \leqslant j<|s|$, можно выбирать произвольно, с тем ограничением, что $\delta_{0}(j), 0 \leqslant j<|s|$, обратимы.

ДокАзАТЕЛьство. Докажем только пункт 1 утверждения; пункт 2 доказывается аналогично. Поскольку старший коэффициент $\alpha_{r}$ обратим, матрица $\mathcal{L}$ обратима и ее обратная $\mathcal{L}^{-1}$ принадлежит $L T_{-r}$, причем старший коэффициент обратной матрицы есть $\Lambda^{-r k} \alpha_{r}^{-1} \Lambda^{r k}$. Поэтому можно ограничиться случаем $r>0$. Искомая матрица $U$ должна удовлетворять уравнению $\mathcal{L} U=U \Lambda^{k r}$, которое сводится к следующим уравнениям на коэффициенты $\beta_{t}$ оператора $U$ :

$$
\beta_{t}=\sum_{i=t}^{0} \alpha_{t+r-i} \Lambda^{k(t+r-i)} \beta_{i} \Lambda^{-k(t+r-i)}, \quad t=0,1, \ldots
$$

Если все $\beta_{i}=\operatorname{diag}\left(\beta_{i}(j)\right)$ и $\alpha_{i}=\operatorname{diag}\left(\alpha_{i}(j)\right)$, то из этого уравнения при $t=0$ следует, что при всех $j \in \mathbb{Z}$

$$
\beta_{0}(j)=\alpha_{r}(j) \beta_{0}(j+r) .
$$

Мы видим, что для решения этого уравнения можно выбрать произвольные обратимые элементы $\beta_{0}(j), 0 \leqslant j<r$, и тогда уравнение (3) определяет элементы $\beta_{0} \in \mathcal{D}_{k}(R)$ единственным образом. Коль скоро мы показали, как найти решение для $\beta_{0}$, можно считать, что мы нашли все $\beta_{l}$ при $l>t$. Блоки следующего коэффициента $\beta_{t}$ оператора $U$ должны удовлетворять соотношениям

$$
\beta_{t}(j)-\alpha_{r}(j) \beta_{t}(j+r)=\sum_{0 \leqslant i<t} \alpha_{t+r-i}(j) \beta_{i}(j+r+t-i),
$$

из которых видно, что в выборе $\beta_{t}(j), 0 \leqslant j<r$, снова имеется свобода, но остальные блоки определяются этим выбором единственным образом. Таким способом мы находим оператор $U$, удовлетворяющий уравнению $\mathcal{L} U=U \Lambda^{k r}$. Этим завершается доказательство утверждения.

\section{3. УРАВНЕНИЯ ЛАКСА В $L T$ И $U T$}

Начнем с коммутативной подалгебры $\mathbf{h}$ в $g l_{k}(\mathbb{C})$, которая определяет вид коммутирующих потоков, лежащих в основе уравнений иерархий. Для максимизации числа коммутирующих потоков будем предполагать $\mathbf{h}$ максимальной. Мы предлагаем читателю два примера максимальных коммутативных алгебр: алгебру диагональных матриц и алгебру

$$
\mathbf{h}=\left\{h=\sum_{i=0}^{k-1} a_{i} B^{i}, \quad \text { где } B=\left(\begin{array}{ccccc}
0 & 1 & 0 & \cdots & 0 \\
\vdots & \ddots & \ddots & \ddots & \vdots \\
\vdots & & \ddots & \ddots & 0 \\
\vdots & & & \ddots & 1 \\
0 & \ldots & \ldots & \cdots & 0
\end{array}\right)\right\},
$$


которые представляют собой предельные случаи соответственно с максимальной и минимальной полупростой частью. Выберем базис $\left\{E_{\alpha} \mid 1 \leqslant \alpha \leqslant m\right\}$ в h. Иерархия, на которой мы сконцентрируем свое внимание, является комбинацией двух других: нижнетреугольной и верхнетреугольной иерархий Тоды. Сначала рассмотрим деформации внутри коммутативной подалгебры $L T(R)$ в $M_{\mathbb{Z}}(\mathbb{C})$, порожденной элементами $\Lambda^{k}$ и $i_{k}\left(E_{\alpha}\right), 1 \leqslant \alpha \leqslant m$. Конкретнее, в $L T(R)$ рассмотрим матрицы

$$
\begin{aligned}
\mathcal{L} & :=\sum_{i \leqslant 1} l_{i} \Lambda^{k i}, & \text { где } \quad l_{1}=\mathrm{Id}=\Lambda^{0}, \\
U_{\alpha} & =\sum_{i \leqslant 0} u_{i, \alpha} \Lambda^{k i}, & \text { где } \quad u_{0, \alpha}=i_{k}\left(E_{\alpha}\right) .
\end{aligned}
$$

Кроме условия $\left[\Lambda^{k}, i_{k}\left(E_{\alpha}\right)\right]=0$, выполнено также соотношение

$$
E_{\alpha} E_{\beta}=\sum_{\gamma} C_{\alpha \beta}^{\gamma} E_{\gamma}
$$

поэтому мы потребуем, чтобы деформации из (6) удовлетворяли равенствам

$$
\left[\mathcal{L}, U_{\alpha}\right]=0, \quad U_{\alpha} U_{\beta}=\sum_{\gamma} C_{\alpha \beta}^{\gamma} U_{\gamma}
$$

для всех индексов. Заметим, что в силу утверждения 2.1 оператор $\mathcal{L}$ можно записать как $\mathcal{L}=U \Lambda^{k} U^{-1}$, где $U \in L T_{0}$ имеет вид $U=\mathrm{Id}+\sum_{i<0} \beta_{i} \Lambda^{k i}$. Если выбрать $U_{\alpha}:=U i_{k}\left(E_{\alpha}\right) U^{-1}$, то уравнения $(7)$ тривиально удовлетворяются. Кроме сохранения алгебраических соотношений, все коэффициенты матриц $\mathcal{L}$ и $U_{\alpha}, \alpha=1, \ldots, m$, также должны принадлежать кольцу функций $R$ от параметров деформации $t_{i \alpha}$, соответствующих коммутирующим направлениям

$$
\Lambda^{i k} i_{k}\left(E_{\alpha}\right), \quad i=0,1, \ldots, \quad \alpha=1, \ldots, m .
$$

Мы предполагаем, что кольцо $R$ устойчиво относительно дифференцирования по $t_{i \alpha}$, а матрица $\mathcal{L}$ должна удовлетворять нелинейным уравнениям

$$
\frac{\partial}{\partial t_{i \alpha}} \mathcal{L}:=\partial_{i \alpha} \mathcal{L}=\left[\left(\mathcal{L}^{i} U_{\alpha}\right)_{+}, \mathcal{L}\right], \quad \partial_{i \alpha} U_{\beta}=\left[\left(\mathcal{L}^{i} U_{\alpha}\right)_{+}, U_{\beta}\right] .
$$

Уравнения (8) называются уравнениями Лакса нижнетреугольной иерархии Тодъ, связанной $c \mathbf{h}$. Из этих уравнений следует, что $\mathcal{L}$ и $U_{\alpha}$ получены путем сопряжения как $\Lambda^{k}$, так и $i_{k}\left(E_{\alpha}\right)$ с помощью одной и той же матрицы, т.е. посредством одевания тривиального решения.

Заметим, что полученная выше форма иерархии аналогична форме КП-иерархии и иерархии других солитонных уравнений, в которых возмущение коммутативной алгебры дифференциальных операторов, соответствующих тривиальному решению, берется внутри кольца псевдодифференциальных операторов (см. [5], [6]).

Уравнения Лакса (8) можно получить как условия совместности для подходящей линейной системы, так называемой линеаризащии иерархии. Пространство, на котором рассматривается такая линеаризация, определяет вид матрицы, которую следует использовать для одевания тривиального решения. Пусть временно $\delta \in M_{\mathbb{Z}}(\mathbb{C})-$ 
матрица, коммутирующая с $\Lambda^{k}$ и $i_{k}\left(E_{\alpha}\right)$. Соответствующий выбор элементов $\delta$ мы обсудим ниже. Теперь свободный левый $L T(R)$-модуль $M^{(\infty)}(\delta)$ осциллирующих матриц типа $\delta$ с сингулярностью не сильнее, чем полюс на бесконечности состоит из элементов, имеющих вид формального произведения элемента из $L T(R)$, матрицы $\delta$ и экспоненциального множителя

$$
\varphi_{\infty}:=\exp \left\{\sum_{i=0}^{\infty} \sum_{\alpha=1}^{m} t_{i \alpha} i_{k}\left(E_{\alpha}\right) \Lambda^{i k}\right\} .
$$

Пусть $\partial_{i \alpha}$ действует на элементы из $L T(R)$ покоэффициентно. На элементе $\varphi:=$ $\widehat{\varphi} \delta \varphi_{\infty} \in M^{(\infty)}(\delta)$ действие $\partial_{i \alpha}$ определяется уравнением

$$
\partial_{i \alpha}\left(\widehat{\varphi} \delta \varphi_{\infty}\right):=\left\{\partial_{i \alpha} \widehat{\varphi}+\widehat{\varphi} \Lambda^{i k} i_{k}\left(E_{\alpha}\right)\right\} \delta \varphi_{\infty}
$$

Заметим, что, поскольку $\delta$ коммутирует с $\Lambda^{k}$ и $i_{k}\left(E_{\alpha}\right)$, умножение на эти матрицы справа хорошо определено на $M^{(\infty)}(\delta)$. Тогда линеаризация состоит из следующей системы уравнений для некоторого $\varphi:=\widehat{\varphi} \delta \varphi_{\infty} \in M^{(\infty)}(\delta)$, где $\widehat{\varphi}=\sum_{i \geqslant 0} w_{i} \Lambda^{-i k}$ при $w_{0}=\mathrm{Id}:$

$$
\mathcal{L} \varphi=\varphi \Lambda^{k}, \quad U_{\alpha} \varphi=\varphi i_{k}\left(E_{\alpha}\right), \quad \partial_{i \alpha} \varphi=\left(\mathcal{L}^{i} U_{\alpha}\right)_{+} \varphi .
$$

Генератор $\delta \varphi_{\infty} \quad L T(R)$-модуля $M^{(\infty)}(\delta)$ удовлетворяет этим уравнениям для тривиального решения $\mathcal{L}=\Lambda^{k}$ и $U_{\alpha}=i_{k}\left(E_{\alpha}\right)$. Другие $\varphi$, удовлетворяющие уравнениям (9), можно, таким образом, рассматривать как возмущения этого тривиального решения. Прежде всего, из первых двух уравнений в (9) следует, что

$$
\mathcal{L}=: \mathcal{L}(\widehat{\varphi})=\widehat{\varphi} \Lambda^{k} \widehat{\varphi}^{-1}, \quad U_{\alpha}=: U_{\alpha}(\widehat{\varphi})=\widehat{\varphi} i_{k}\left(E_{\alpha}\right) \widehat{\varphi}^{-1} .
$$

Отсюда

$$
\partial_{i \alpha} \mathcal{L}=\left[\partial_{i \alpha} \widehat{\varphi} \cdot \widehat{\varphi}^{-1}, \mathcal{L}\right], \quad \partial_{i \alpha} U_{\beta}=\left[\partial_{i \alpha} \widehat{\varphi} \cdot \widehat{\varphi}^{-1}, U_{\beta}\right] .
$$

Теперь третье уравнение в (9) дает

$$
\partial_{i \alpha} \widehat{\varphi} \cdot \widehat{\varphi}^{-1}+\widehat{\varphi} \Lambda^{i k} i_{k}\left(E_{\alpha}\right) \widehat{\varphi}^{-1}=\left(\mathcal{L}^{i} U_{\alpha}\right)_{+}
$$

В частности, мы видим, что

$$
\partial_{i \alpha} \widehat{\varphi} \cdot \widehat{\varphi}^{-1}=-\left(\mathcal{L}^{i} U_{\alpha}\right)_{-}=\left(\mathcal{L}^{i} U_{\alpha}\right)_{+}-\mathcal{L}^{i} U_{\alpha}
$$

и, подставляя это равенство в уравнение (10), получаем уравнения Лакса нижнетреугольной иерархии Тоды для $\mathcal{L}(\widehat{\varphi})$ и $U_{\alpha}(\widehat{\varphi}), \alpha=1, \ldots, m$. Мы будем говорить, что $\varphi$ является волновой матрицей типа $\delta$ на бесконечности для такого набора решений. Достаточно доказать более слабый вариант линеаризации.

ЛЕмма 3.1. Если для $\varphi:=\widehat{\varphi} \delta \varphi_{\infty} \in M^{(\infty)}(\delta)$, где $\widehat{\varphi}=\sum_{i \geqslant 0} w_{i} \Lambda^{-i k} n p u w_{0}=\mathrm{Id}$, для всех $\alpha$ и в выполнено соотношение

$$
\partial_{i \alpha} \varphi=P_{i \alpha} \varphi, \quad \text { əде } \quad P_{i \alpha} \in U T_{0}(R) \cap L T(R),
$$

то $P_{i \alpha}=\left(\mathcal{L}(\widehat{\varphi})^{i} U_{\alpha}(\widehat{\varphi})\right)_{+}$, а $\varphi$ является волновой матрицей типа $\delta$ на бесконечности для $\mathcal{L}(\widehat{\varphi})$ и $U_{\alpha}(\widehat{\varphi}), \alpha=1, \ldots, m$. 
Доказательство. Матрица $P_{i \alpha}$ как раз равна левой части уравнения $(11)$, и взятие верхнетреугольной части дает искомое тождество $P_{i \alpha}=\left(\mathcal{L}(\widehat{\varphi})^{i} U_{\alpha}(\widehat{\varphi})\right)_{+}$.

Для верхнетреугольной иерархии мы будем действовать аналогично, но рассматривать лежащие внутри $U T(R)$ деформации коммутативной алгебры, порожденной элементами $\Lambda^{-k}$ и $i_{k}\left(F_{\beta}\right), 1 \leqslant \beta \leqslant m$, где $\left\{F_{\beta} \mid 1 \leqslant \beta \leqslant m\right\}$ представляет собой некоторый базис в $\mathbf{h}$ с законом умножения

$$
F_{\alpha} F_{\beta}=\sum_{\gamma} D_{\alpha \beta}^{\gamma} F_{\gamma}
$$

Деформациями элементов $\Lambda^{-k}$ (элементов $\left.i_{k}\left(F_{\beta}\right)\right)$ являются матрицы $\mathcal{M}$ (соответственно $\left.V_{\beta}\right)$ вида

$$
\mathcal{M}:=\sum_{i \geqslant-1} m_{i} \Lambda^{k i}, \quad V_{\beta}:=\sum_{i \geqslant 0} v_{i, \beta} \Lambda^{k i}, \quad \beta=1, \ldots, m,
$$

причем старший коэффициент $\mathcal{M}$ обратим, а старший коэффициент $V_{\beta}$ равен $w_{0} i_{k}\left(F_{\beta}\right) w_{0}^{-1}$ для некоторого обратимого $w_{0}$, удовлетворяющего соотношению

$$
m_{-1}=w_{0} \Lambda^{-k} w_{0}^{-1} \Lambda^{k}
$$

Существование такого $w_{0}$ следует из доказательства утверждения 2.1. Как и ранее, алгебраические соотношения между исходными матрицами должны сохраняться при их деформациях, т.е. должны иметь место равенства

$$
\left[\mathcal{M}, V_{\beta}\right]=0, \quad V_{\alpha} V_{\beta}=\sum_{\gamma} D_{\alpha \beta}^{\gamma} V_{\gamma}
$$

Приведем примеры деформаций, удовлетворяющих этим уравнениям:

$$
\mathcal{M}:=V \Lambda^{-k} V^{-1}, \quad V_{\beta}=V i_{k}\left(F_{\beta}\right) V^{-1}, \quad V=\sum_{i \geqslant 0} \delta_{i} \Lambda^{i k}, \quad \text { где } \quad \delta_{0} \quad \text { обратим. }
$$

Коэффициенты матриц $\mathcal{M}$ и $V_{\alpha}$ должны принадлежать кольцу функций $R$ от параметров деформации $s_{j \beta}$, соответствующих коммутирующим направлениям

$$
\Lambda^{-j k} i_{k}\left(F_{\beta}\right), \quad j=1,2, \ldots, \quad \beta=1, \ldots, m .
$$

Мы предполагаем, что кольцо $R$ устойчиво относительно дифференцирования по $s_{j \beta}$, а деформированные матрицы должны удовлетворять нелинейным уравнениям

$$
\frac{\partial}{\partial s_{j \beta}} \mathcal{M}=: \partial_{j \beta} \mathcal{M}=\left[\left(\mathcal{M}^{j} V_{\beta}\right)_{-}, \mathcal{M}\right], \quad \partial_{j \beta} V_{\alpha}=\left[\left(\mathcal{M}^{j} V_{\beta}\right)_{-}, V_{\alpha}\right] .
$$

Уравнения (13) называются уравнениями Лакса верхнетреугольной иерархии Тодь, свлзанной $c \mathbf{h}$, причем из них следует, что $\mathcal{M}$ и $V_{\alpha}$ получаются путем сопряжения как $\Lambda^{-k}$, так и $i_{k}\left(F_{\alpha}\right)$ с помощью одной и той же матрицы, т.е. путем одевания тривиального решения. 
Как и ранее, искомые уравнения Лакса можно получить как условия совместности для подходящей линейной системы, также называемой линеаризацией иерархии. Матрица, с помощью которой сопрягается тривиальное решение, встроена в пространство, на котором реализована линеаризация. В данном случае мы также начнем с матрицы $\delta \in M_{\mathbb{Z}}(\mathbb{C})$, коммутирующей с $\Lambda^{-k}$ и $i_{k}\left(F_{\beta}\right)$, и рассмотрим свободный левый $U T(R)$-модуль $M^{(0)}(\delta)$ осциллирующих матриц типа $\delta$ с сингулярностью не сильнее, чем полюс в нуле, состоящий из элементов, имеющих вид формального произведения элементов из $U T(R)$, матрицы $\delta$ и экспоненциального множителя:

$$
\psi_{0}:=\exp \left\{\sum_{j=1}^{\infty} \sum_{\beta=1}^{m} s_{j \beta} i_{k}\left(F_{\beta}\right) \Lambda^{-j k}\right\} .
$$

Аналогично, пусть $\partial_{j \beta}$ действуют на элементы из $U T(R)$ покоэффициентно. Действие $\partial_{j \beta}$ на элемент $\psi:=\widehat{\psi} \delta \psi_{0} \in M^{(0)}(\delta)$ определяется как

$$
\partial_{j \beta}\left(\widehat{\psi} \delta \psi_{0}\right):=\left\{\partial_{j \beta} \widehat{\psi}+\widehat{\psi} \Lambda^{-j k} i_{k}\left(F_{\beta}\right)\right\} \delta \psi_{0}
$$

Поскольку $\delta$ коммутирует с $\Lambda^{-k}$ и $i_{k}\left(F_{\alpha}\right)$, умножение на эти матрицы справа является хорошо определенным оператором на $M^{(0)}(\delta)$. Тогда линеаризация состоит из следующей системы уравнений для $\psi:=\widehat{\psi} \delta \psi_{0} \in M^{(0)}(\delta)$, где $\widehat{\psi}=\sum_{i \geqslant 0} w_{i} \Lambda^{i k}$ с обратимым $w_{0}$ :

$$
\mathcal{M} \psi=\psi \Lambda^{-k}, \quad V_{\alpha} \psi=\psi i_{k}\left(F_{\alpha}\right), \quad \partial_{j \beta} \psi=\left(\mathcal{M}^{j} V_{\beta}\right)_{-} \psi .
$$

Можно считать, что $\psi$, удовлетворяющие уравнениям (14), являются возмущениями генератора $\delta \psi_{0} \quad U T(R)$-модуля $M^{(0)}(\delta)$. Генератор $\delta \psi_{0}$ удовлетворяет этим уравнениям для тривиального решения $\mathcal{M}=\Lambda^{-k}, V_{\alpha}=i_{k}\left(F_{\alpha}\right)$. Из первых двух уравнений в (14) снова следует, что матрицы $\mathcal{M}$ и $V_{\alpha}$ определяются возмущающим множителем $\widehat{\psi}$, потому что они получены путем сопряжения тривиального решения с помощью множителя:

$$
\mathcal{M}=\mathcal{M}(\widehat{\psi}):=\widehat{\psi} \Lambda^{-k} \widehat{\psi}^{-1}, \quad V_{\alpha}=V_{\alpha}(\widehat{\psi}):=\widehat{\psi} i_{k}\left(F_{\alpha}\right) \hat{\psi}^{-1}
$$

Поскольку матрицы $\mathcal{M}$ и $V_{\alpha}$ получаются путем одевания тривиального решения с помощью $\widehat{\psi}$, имеем

$$
\partial_{j \beta} \mathcal{M}=\left[\partial_{j \beta} \widehat{\psi} \cdot \widehat{\psi}^{-1}, \mathcal{M}\right], \quad \partial_{j \beta} V_{\alpha}=\left[\partial_{j \beta} \widehat{\psi} \cdot \widehat{\psi}^{-1}, V_{\alpha}\right] .
$$

Теперь третье из уравнений (14) дает

$$
\partial_{j \beta} \widehat{\psi} \cdot \widehat{\psi}^{-1}+\widehat{\psi} \Lambda^{-j k} i_{k}\left(F_{\beta}\right) \widehat{\psi}^{-1}=\left(\mathcal{M}^{j} V_{\beta}\right)_{-} .
$$

В частности, мы видим, что

$$
\partial_{j \beta} \widehat{\psi} \cdot \widehat{\psi}^{-1}=-\left(\mathcal{M}^{j} V_{\alpha}\right)_{+}=\left(\mathcal{M}^{j} V_{\beta}\right)_{-}-\mathcal{M}^{j} V_{\alpha}
$$

и, подставляя это соотношение в уравнение (15), получаем в результате уравнения Лакса верхнетреугольной иерархии Тоды для $\mathcal{M}(\widehat{\psi})$ и $V_{\alpha}(\widehat{\psi}), \alpha=1, \ldots, m$. Мы будем говорить, что для этого набора решений $\psi$ является волновой матрищей типа $\delta$ в нуле. Аналогично лемме 3.1, достаточно показать следующее. 
Лемма 3.2. Предположим, что $\psi:=\widehat{\psi} \delta \psi_{0} \in M^{(0)}(\delta)$, где $\widehat{\psi}=\sum_{i \geqslant 0} w_{i} \Lambda^{i k}$ с обратимым $w_{0}$, причем для всех $\beta$ и ј имеет место

$$
\partial_{j \beta}(\psi)=Q_{j \beta} \psi, \quad \text { əде } \quad Q_{j \beta} \in L T_{-1}(R) \cap U T(R) .
$$

Тогда $Q_{j \beta}=\left(\mathcal{M}(\widehat{\psi})^{j} V_{\beta}(\widehat{\psi})\right)_{-}$, а $\psi$ является волновой матрицей типа $\delta$ в нуле для матрии, $\mathcal{M}(\widehat{\psi})$ u $V_{\beta}(\widehat{\psi}), \beta=1, \ldots, m$.

Следующий шаг состоит в комбинировании обеих иерархий; таким образом мы одновременно рассматриваем деформации $\left\{\mathcal{L}, U_{\gamma}\right\}$ и $\left\{\mathcal{M}, V_{\sigma}\right\}$, удовлетворяющие в первую очередь уравнениям (7), (8), (12), (13). Однако теперь мы предполагаем, что коэффициенты этих деформаций зависят как от $t_{i \alpha}$, так и от $s_{j \beta}$, и что зависимость от дополнительных параметров задается формулами

$$
\begin{aligned}
\partial_{j \beta} \mathcal{L} & =\left[\left(\mathcal{M}^{j} V_{\beta}\right)_{-}, \mathcal{L}\right], & & \partial_{j \beta} U_{\gamma}=\left[\left(\mathcal{M}^{j} V_{\beta}\right)_{-}, U_{\gamma}\right], \\
\partial_{i \alpha} \mathcal{M} & =\left[\left(\mathcal{L}^{i} U_{\alpha}\right)_{+}, \mathcal{M}\right], & \partial_{i \alpha} V_{\sigma} & =\left[\left(\mathcal{L}^{i} U_{\alpha}\right)_{+}, V_{\sigma}\right] .
\end{aligned}
$$

Будем говорить, что уравнения (8), (13), (16), (17) суть уравнения Лакса для двумерной бесконечной иерархии Тодь, связанной $c \mathbf{h}$. Подходящим образом комбинируя линеаризации обеих деформаций, эти уравнения также можно рассматривать как условие совместности этих систем. Как на $L T(R)$, так и на $U T(R)$ частные производные по параметрам деформации берутся покоэффициентно. Определим действие оператора $\partial_{j \beta}$ на элементы из $M^{(\infty)}(\delta)$ как

$$
\partial_{j \beta}\left(\widehat{\varphi} \delta \varphi_{\infty}\right)=\partial_{j \beta} \widehat{\varphi} \cdot \delta \varphi_{\infty}
$$

и действие $\partial_{i \alpha}$ на $M^{(0)}(\delta)-$ как

$$
\partial_{i \alpha}\left(\widehat{\psi} \delta \psi_{0}\right)=\partial_{i \alpha} \widehat{\psi} \cdot \delta \psi_{0}
$$

Линеаризация двумерной бесконечной иерархии Тоды, связанной $\mathbf{c ~ h}$, состоит тогда из уравнений

$$
\begin{aligned}
\mathcal{L} \varphi & =\varphi \Lambda^{k}, & U_{\gamma} \varphi & =\varphi i_{k}\left(E_{\gamma}\right), \\
\partial_{i \alpha} \varphi & =\left(\mathcal{L}^{i} U_{\alpha}\right)_{+} \varphi, & \partial_{j \beta} \varphi & =\left(\mathcal{M}^{j} V_{\beta}\right)_{-} \varphi ; \\
\mathcal{M} \psi & =\psi \Lambda^{-k}, & V_{\sigma} \psi & =\psi i_{k}\left(F_{\sigma}\right), \\
\partial_{i \alpha} \psi & =\left(\mathcal{L}^{i} U_{\alpha}\right)_{+} \psi, & \partial_{j \beta} \psi & =\left(\mathcal{M}^{j} V_{\beta}\right)_{-} \psi
\end{aligned}
$$

для пары $(\varphi, \psi) \in M^{(\infty)}(\delta) \times M^{(0)}(\delta)$ вида $\widehat{\varphi}=\sum_{i \geqslant 0} w_{i} \Lambda^{-i k}$, где $w_{0}=\operatorname{Id}$ и $\widehat{\psi}=$ $\sum_{i \geqslant 0} d_{i} \Lambda^{i k}$ с обратимым $d_{0}$. Как и выше, мы покажем, что деформации $\mathcal{L}(\widehat{\varphi}), U_{\gamma}(\widehat{\varphi})$ (деформации $\left.\mathcal{M}(\psi), V_{\sigma}(\psi)\right)$ являются решениями двумерной иерархии Тоды, связанной с h. Пара $(\varphi, \psi)$ называется парой волновых матрии типа $\delta$ для двумерной бесконечной иерархии Тодь, связанной с $\mathbf{h}$. Леммы 3.1 и 3.2 объединяются в следующую лемму. 
Лемма 3.3. Рассмотрим пару $(\varphi, \psi)$ в пространстве $M^{(\infty)}(\delta) \times M^{(0)}(\delta)$ вида $\widehat{\varphi}=\sum_{i \geqslant 0} w_{i} \Lambda^{-i k}$, где $w_{0}=\mathrm{Id}, u \widehat{\psi}=\sum_{i \geqslant 0} d_{i} \Lambda^{i k}$ c обратимым $d_{0}$. Полагаем, что для всех $\alpha, i, \beta$ и в выполнено

$$
\begin{aligned}
\partial_{i \alpha} \varphi & =P_{i \alpha} \varphi, & \partial_{j \beta} \varphi & =Q_{j \beta} \varphi, \\
\partial_{j \beta} \psi & =Q_{j \beta} \psi, & \partial_{i \alpha} \psi & =P_{i \alpha} \psi,
\end{aligned}
$$

где $P_{i \alpha} \in U T_{0}(R) \cap L T(R)$ u $Q_{j \beta} \in L T_{-1}(R) \cap U T(R)$. Тогда $P_{i \alpha}=\left(\mathcal{L}(\widehat{\varphi})^{i} U_{\alpha}(\widehat{\varphi})\right)_{+}$, $Q_{j \beta}=\left(\mathcal{M}(\widehat{\psi})^{j} V_{\beta}(\widehat{\psi})\right)_{-}$, а пара $(\varphi, \psi)$ является парой волновых матриц, типа $\delta$ для матрии, $\mathcal{L}(\widehat{\varphi}), \mathcal{M}(\widehat{\psi}), U_{\gamma}(\widehat{\varphi})$ u $V_{\sigma}(\widehat{\psi})$.

\section{4. ПОСТРОЕНИЕ РЕШЕНИЙ ИЕРАРХИИ}

Пусть $S^{1}$ - единичная окружность в комплексной плоскости. Конкретные элементы $M^{(\infty)}(\delta)$ и $M^{(0)}(\delta)$ можно получить как $(\mathbb{Z} \times \mathbb{Z})$-матрицы, соответствующие подходящим ограниченным операторам в гильбертовом пространстве $H=L^{2}\left(S^{1}, \mathbb{C}^{k}\right)$. Элементы пространства $H$ обозначаются

$$
h=\sum_{n \in \mathbb{Z}} a(n) z^{n}, \quad \text { где } \quad a(n) \in \mathbb{C}^{k} \quad \text { для всех } n \in \mathbb{Z} .
$$

Пусть $(\cdot \mid \cdot)$ - стандартное скалярное произведение на $\mathbb{C}^{k}$. Скалярное произведение на $H$ тогда дается соотношением

$$
\left\langle\sum_{n \in \mathbb{Z}} a(n) z^{n} \mid \sum_{n \in \mathbb{Z}} b(n) z^{n}\right\rangle:=\sum_{n \in \mathbb{Z}}(a(n) \mid b(n)) .
$$

Если $\left\{f_{i} \mid 0 \leqslant i \leqslant k-1\right\}$ обозначает стандартный базис пространства $\mathbb{C}^{k}$, где $f_{i}$ таков, что его $(i+1)$-й элемент равен единице, а остальные элементы равны нулю, то мы получаем ортонормированный базис $\left\{e_{i} \mid i \in \mathbb{Z}\right\}$ в $H$, положив $e_{s+k j}:=f_{s} z^{j}$ для всех $j \in \mathbb{Z}$ и всех $s, 0 \leqslant s \leqslant k-1$.

Элемент $h=\sum \alpha_{i} e_{i}$ также отождествляется с $(\mathbb{Z} \times 1)$-матрицей

$$
h=\left(\ldots, \alpha_{i-1}, \alpha_{i}, \alpha_{i+1}, \ldots\right)^{\mathrm{T}},
$$

где верхний индекс Т обозначает транспонирование матрицы. Следовательно, действие ограниченного оператора $B \in B(H)$ соответствует в матричном представлении умножению слева на комплексную $(\mathbb{Z} \times \mathbb{Z})$-матрицу $[B]=\left([B]_{l k}\right)$, где

$$
[B]_{l k}=\left\langle B\left(e_{k}\right) \mid e_{l}\right\rangle
$$

Базисные матрицы $E_{(i, j)}$ соответствуют операторам $\mathcal{E}_{(i, j)} \in B(H), i, j \in \mathbb{Z}$. Внутри $H$ рассмотрим некоторое множество подпространств. Для каждого $i \in \mathbb{Z}$ обозначим $H^{(i)}$ комплексное подпространство в $H$, линейно порожденное элементами $f_{s} z^{i}, s=$ $0,1, \ldots, k-1$. Проекция $H \mapsto H^{(i)}$, задаваемая с помощью $\sum_{j \in \mathbb{Z}} h(j) z^{j} \mapsto h(i) z^{i}$, обозначается как $p^{(i)}$. Пространство $H$ разлагается в прямую сумму $H=\bigoplus_{i \in \mathbb{Z}} H^{(i)}$, 
и это разложение определяет для каждого ограниченного линейного оператора $B \in$ $B(H)$ соответствующий блок разложения,

$$
B=\left(\begin{array}{ccccc}
\ddots & \vdots & \vdots & \vdots & . \\
\ldots & B_{n-1 n-1} & B_{n-1 n} & B_{n-1 n+1} & \ldots \\
\ldots & B_{n n-1} & B_{n n} & B_{n n+1} & \ldots \\
\ldots & B_{n+1 n-1} & B_{n+1 n} & B_{n+1 n+1} & \ldots \\
. & \vdots & \vdots & \vdots & \ddots
\end{array}\right),
$$

где $B_{i j}:=p^{(i)} \circ B \mid H^{(j)}$, и разложение матрицы $[B]=\left(\left[B_{i j}\right]\right)$ по $(k \times k)$-блокам. Другое важное для дальнейших рассуждений подпространство - это подпространство $H_{j}, j \in \mathbb{Z}$, определяемое как $H_{j}=\bigoplus_{i \geqslant j} H^{(i)}$; отвечающая ему ортогональная проекция есть $p_{j}:=\bigoplus_{i \geqslant j} p^{(i)}$. Мы используем специальное обозначение для разложения любого элемента $b \in B(H)$ в соответствии с разбиением $H=H_{j}^{\perp} \oplus H_{j}$, а именно

$$
b=\left(\begin{array}{ll}
b_{--}(j) & b_{-+}(j) \\
b_{+-}(j) & b_{++}(j)
\end{array}\right)
$$

Далее рассмотрим группу, являющуюся отправной точкой построения пары волновых матриц для двумерной иерархии Тоды. Эта группа выбирается так, чтобы коэффициенты решения можно было выразить в терминах некоторых определителей, см. [7]. Рассмотрим множество операторов

$$
\operatorname{Id}+\mathcal{N}=\{\operatorname{Id}+A \mid A: H \mapsto H-\text { ядерный оператор }\}
$$

с метрикой, определяемой как

$$
\left\|\left(\mathrm{Id}+A_{1}\right)-\left(\operatorname{Id}+A_{2}\right)\right\|_{1}=\left\|A_{1}-A_{2}\right\|_{1},
$$

где $\|\cdot\|_{1}$ обозначает следовую норму на $\mathcal{N}$. Напомним, что [8] любой оператор вида $\mathrm{Id}+A$ обратим, если и только если $\operatorname{det}(\mathrm{Id}+A) \neq 0$, и что обратный оператор также принадлежит к классу $\mathrm{Id}+\mathcal{N}$. Поэтому подмножество $G_{1}$, определяемое как

$$
G_{1}=\{g \in \mathrm{GL}(H) \cap \mathrm{Id}+\mathcal{N}\},
$$

является банаховой группой Ли с алгеброй Ли $\mathcal{G}_{1}$, задаваемой пространством ядерных операторов на $H$. Алгебра Ли $\mathcal{G}_{1}$ есть сумма следующих подалгебр Ли:

$$
\mathcal{P}_{1}:=\left\{p=\left(p_{i j}\right) \in \mathcal{G}_{1} \mid p_{i j}=0 \text { для всех } i>j\right\}
$$

и

$$
\mathcal{U}_{-}:=\left\{u=\left(u_{i j}\right) \in \mathcal{G}_{1} \mid u_{i j}=0 \quad \text { для всех } i \leqslant j\right\} .
$$

Соответствующие им группы Ли имеют вид

$$
P_{1}:=\left\{p=\left(p_{i j}\right) \in G_{1} \mid p_{i j}=0 \quad \text { для всех } i>j\right\}
$$

И

$$
U_{-}:=\left\{u=\left(u_{i j}\right) \in G \mid \begin{array}{ccc}
u_{i j}=0 & \text { для всех } & i<j \\
u_{i i}=\mathrm{Id} & \text { для всех } \quad i \in \mathbb{Z}
\end{array}\right\} .
$$


Наборы $(\mathbb{Z} \times \mathbb{Z})$-матриц, отвечающие группам $P_{1}$ и $U_{-}$, обозначаются как $\left[P_{1}\right]$ и $\left[U_{-}\right]$. Поскольку отображение из $\mathcal{U}_{-} \times \mathcal{P}_{1}$ в $G_{1}$, определяемое как $(u, p) \mapsto e^{u} e^{p}$, есть локальный диффеоморфизм в точке $(0,0)$, множество $U_{-} P$ является открытым подмножеством в $G_{1}$. Его можно охарактеризовать так же, как и в конечномерном случае.

УТВЕРЖДЕНИЕ 4.1. Пусть $\Omega \subset G-$ набор всех $g \in G_{1}$ maкuх, что $g_{--}(i)$ является обратимым для всех $i \in \mathbb{Z}$. Тогда $\Omega$ совпадает с $U_{-} P_{1}$ и называется большой клеткой в $G_{1}$.

Форма волновых матриц $\varphi_{0}$ и $\psi_{0}$ позволяет определить коммутирующие потоки, соответствующие двумерной иерархии Тоды, однако ни одна из этих матриц не может появиться как $(\mathbb{Z} \times \mathbb{Z})$-матрица какого-либо оператора из $G_{1}$. При этом они представляют собой естественные компоненты группы коммутирующих потоков, связанных с $\mathbf{h}$, которая вкладывается в $\mathrm{GL}(H)$. Для каждого $r>1$ рассмотрим кольцо

$$
A_{r}=\left\{z \in \mathbb{C}\left|r^{-1} \leqslant\right| z \mid \leqslant r\right\}
$$

вокруг единичной окружности $S^{1}$. Пусть $U$ - любая открытая связная окрестность этого кольца в комплексной плоскости. Тогда будем писать $\Gamma_{r}(U, \mathbf{h})$ для множества голоморфных отображений $\gamma: U \mapsto \mathbf{h}$ таких, что $\operatorname{det}(\gamma(u)) \neq 0$ для всех $u \in U$. Это множество есть банахова группа Ли с соответствующей равномерной сходимостью на $A_{r}$. Ее прямой предел по $r$ обозначим как $\Gamma(\mathbf{h})$. Каждое $\gamma \in \Gamma(\mathbf{h})$ можно разложить в ряд Фурье

$$
\sum_{i \in \mathbb{Z}} \gamma_{i} z^{i}, \quad \text { где } \quad \gamma_{i} \in \mathbf{h} .
$$

Мы получаем непрерывное вложение $\Gamma(\mathbf{h})$ в $\mathrm{GL}(H)$ в соответствии с тем, что $\Gamma(\mathbf{h})$ действует на $H$ умножением слева на этот ряд. Таким образом определен ограниченный оператор $M_{\gamma}: H \mapsto H$. Заметим, что пересечение $G_{1}$ и образа $\Gamma(\mathbf{h})$ равно единице. Если $\gamma(0) \in \Gamma(\mathbf{h})$ обозначает элемент $u \mapsto \operatorname{Id~} u^{-1}$, то матрица $M_{\gamma(0)}$ просто равна $\Lambda$. Умножение слева на матрицу $A \in g l_{k}(\mathbb{C})$ определяет ограниченный оператор $M_{A}$ из $H$, его матрица равна $i_{k}(A)$ и, аналогично случаю $\Gamma(\mathbf{h})$, оператор $M_{A}$ принадлежит алгебре Ли $\mathcal{G}_{1}$, только если $A$ равно нулю. Поскольку все операторы в $B \in B(H)$, коммутирующие с $M_{\gamma(0)}$, можно записать в виде

$$
B=\sum_{i \in \mathbb{Z}} M_{B_{i}} M_{\gamma(0)}^{i}, \quad \text { где } \quad B_{i} \in g l_{k}(\mathbb{C}),
$$

мы видим, что $\Gamma(\mathbf{h})$ содержит большинство направлений, коммутирующих с $M_{\gamma(0)}$ и $M_{A}, A \in \mathbf{h}$. Заметим, что из такого выбора вложения $\Gamma(\mathbf{h})$ в $\operatorname{GL}(H)$ следует, что

$$
\left[M_{\gamma}\right]=\sum_{i \in \mathbb{Z}} i_{k}\left(\gamma_{i}\right) \Lambda^{-k i}
$$

Теперь рассмотрим вид этих потоков подробнее. Пусть $\mathbf{h}_{\mathrm{ss}}-$ подмножество полупростых элементов в $\mathbf{h}$ и пусть $\mathbf{h}_{\mathrm{n}}-$ набор нильпотентных элементов в $\mathbf{h}$. Из того 
факта, что $\mathbf{h}$ является прямой суммой этих подпространств, следует, что группа $\Gamma(\mathbf{h})$ является прямым произведением групп

$$
\Gamma(\mathbf{h})_{\mathrm{ss}}:=\left\{\gamma \mid \gamma \in \Gamma(\mathbf{h}), \gamma(u) \in \mathbf{h}_{\mathrm{ss}} \quad \text { для всех } u\right\}
$$

и

$$
\Gamma(\mathbf{h})_{\mathrm{u}}:=\{\gamma \mid \gamma \in \Gamma(\mathbf{h}), \gamma(u) \text { является унипотентной для всех } u\} .
$$

Теперь легко видеть, что любое $\gamma \in \Gamma(\mathbf{h})_{\text {u }}$ можно записать в виде

$$
\gamma=\exp \left(\sum_{s \in \mathbb{Z}} k_{s} z^{s}\right)=\exp \left(\sum_{s>0} k_{s} z^{s}\right) \exp \left(\sum_{s \leqslant 0} k_{s} z^{s}\right),
$$

где $k_{s} \in \mathbf{h}_{\mathrm{n}}$ для всех $s \in \mathbb{Z}$. Это показывает, что элементы $\Gamma(\mathbf{h})_{\text {u }}$ разлагаются в точности на элементы, которые имеют аналитическое продолжение во внутренность $S^{1}$, и элементы, которые голоморфно продолжаются на “бесконечности".

Как и в случае группы $\Gamma(\mathbf{h})_{\mathrm{ss}}$, напомним, что если $U$ - открытая связная окрестность окружности $S^{1}$, то любое голоморфное отображение $f: U \mapsto \mathbb{C}^{*}$ разлагается как

$$
f(z)=\left\{1+\sum_{i>0} b_{i} z^{i}\right\} z^{m}\left\{\sum_{j \leqslant 0} c_{j} z^{j}\right\}, \quad \text { где } \quad c_{0} \neq 0 \quad \text { и } \quad m \in \mathbb{Z} .
$$

Применяя это к группе $\Gamma(\mathbf{h})_{\mathrm{ss}}$, мы приходим к следующему разложению для $\Gamma(\mathbf{h})$.

УТВЕРЖДЕНИЕ 4.2. Существует подгруппа $\Delta(\mathbf{h})$ в $\Gamma(\mathbf{h})_{\mathrm{ss}}$, изоморфная $\mathbb{Z}^{r}$, где $r$ - размерность $\mathbf{h}_{\mathrm{ss}}$, такая, что $\Gamma(\mathbf{h})=\Gamma(\mathbf{h})_{+} \Delta(\mathbf{h}) \Gamma(\mathbf{h})_{-}$,

$$
\begin{aligned}
& \Gamma(\mathbf{h})_{+}=\left\{\gamma \mid \gamma=\exp \left(\sum_{s \leqslant 0} \gamma_{s} z^{s}\right), \quad \gamma_{s} \in \mathbf{h} \quad \text { для всех } \quad s \leqslant 0\right\}, \\
& \Gamma(\mathbf{h})_{-}=\left\{\gamma \mid \gamma=\exp \left(\sum_{s>0} \gamma_{s} z^{s}\right), \quad \gamma_{s} \in \mathbf{h} \quad \text { для всех } \quad s>0\right\} .
\end{aligned}
$$

В случае, когда $\mathbf{h}$ - диагональная матрица, можно взять

$$
\Delta(\mathbf{h})=\left\{\operatorname{diag}\left(z^{m_{1}}, \ldots, z^{m_{k}}\right) \mid m_{i} \in \mathbb{Z}\right\}
$$

Однако если мы возьмем унипотентную алгебру (5), то группа $\Delta(\mathbf{h})$ сократится до подгруппы в (18), состоящей из элементов, для которых все $m_{i}$ равны некоторому $m \in \mathbb{Z}$.

Если взять базисы $\left\{E_{\alpha} \mid 1 \leqslant \alpha \leqslant m\right\}$ и $\left\{F_{\beta} \mid 1 \leqslant \beta \leqslant m\right\}$ в $\mathbf{h}$, то для каждого элемента $\gamma_{+}$из $\Gamma(\mathbf{h})_{+}$и соответственно $\gamma_{-}$из $\Gamma(\mathbf{h})_{-}$найдется такое $r>1$, что

$$
\begin{aligned}
& \gamma_{+}=\exp \left(\sum_{i=0}^{\infty} \sum_{\alpha=1}^{m} t_{i \alpha} E_{\alpha} z^{-i}\right), \quad t_{i \alpha} \in \mathbb{C}, \quad \sum_{i, \alpha}\left|t_{i \alpha}\right| r^{i}<\infty, \\
& \gamma_{-}=\exp \left(\sum_{j=1}^{\infty} \sum_{\beta=1}^{m} s_{j \beta} F_{\beta} z^{j}\right), \quad s_{j \beta} \in \mathbb{C}, \quad \sum_{j, \beta}\left|s_{j \beta}\right| r^{j}<\infty .
\end{aligned}
$$


Другими словами, $t_{i \alpha}$ представляют собой координаты на $\Gamma(\mathbf{h})+$ по отношению к направлениям $E_{\alpha} z^{-i}, \alpha=1, \ldots, m$, a $s_{j \beta}$ представляют собой координаты на $\Gamma(\mathbf{h})_{-}$по отношению к направлениям $F_{\beta} z^{j}, j=1,2, \ldots, \beta=1, \ldots, m$. Очевидно, что матрица для $\gamma_{+}$совпадает с $\varphi_{0}$, а матрица для $\gamma_{-}-$с $\psi_{0}$, тем самым мы учитываем выражения "в окрестности бесконечности" и "в окрестности нуля", привязанные к волновым матрицам.

Из утверждения 4.2 мы видим, что каждое $\gamma \in \Gamma(\mathbf{h})$ единственным образом может быть представлено в виде $\gamma=\gamma_{+} \delta(\gamma) \gamma_{-}$, где $\gamma_{+} \in \Gamma(\mathbf{h})_{+}, \delta(\gamma) \in \Delta(\mathbf{h})$ и $\gamma_{-} \in \Gamma(\mathbf{h})_{-}$. Поскольку ядерные операторы образуют двусторонний идеал в кольце ограниченных операторов на $H$, группа $\Gamma(\mathbf{h})$ действует сопряжением на группе $G_{1}$. Для каждого $g \in G_{1}$ рассмотрим открытое подмножество в $\Gamma(\mathbf{h})$, определяемое как

$$
\Gamma(g, \mathbf{h})=\left\{\gamma \in \Gamma(\mathbf{h}) \mid \gamma g \gamma^{-1} \in \Omega\right\}
$$

По определению для всех $\gamma \in \Gamma(g, \mathbf{h})$ имеем

$$
\left[\gamma g \gamma^{-1}\right]=\Phi_{-}(\gamma, g)^{-1} \Phi_{+}(\gamma, g)
$$

где $\Phi_{-}(\gamma, g) \in\left[U_{-}\right]$и $\Phi_{+}(\gamma, g) \in\left[P_{1}\right]$. В качестве кольца $R$ возьмем теперь набор голоморфных функций на $\Gamma(g, \mathbf{h})$, тогда $\Phi_{-}(\gamma, g) \in L T_{0}(R)$ и $\Phi_{+}(\gamma, g) \in U T_{0}(R)$. Пусть

$$
\widehat{\Psi}_{-}(\gamma, g):=\Phi_{-}(\gamma, g)\left[\gamma_{-}\right], \quad \widehat{\Psi}_{+}(\gamma, g):=\Phi_{+}(\gamma, g)\left[\gamma_{+}\right] .
$$

Тогда $\widehat{\Psi}_{-}(\gamma, g) \in L T_{0}(R), \widehat{\Psi}_{+}(\gamma, g) \in U T_{0}(R)$. Из уравнения (19) следует, что эти матрицы связаны следующим образом:

$$
\widehat{\Psi}_{-}(\gamma, g)[\delta(\gamma)]\left[\gamma_{+}\right][g]=\widehat{\Psi}_{+}(\gamma, g)[\delta(\gamma)]\left[\gamma_{-}\right],
$$

где произведения матриц в обеих частях равенства хорошо определены, поскольку они соответствуют композиции ограниченных операторов на $H$. Очевидно,

$$
\begin{aligned}
& \Psi_{-}(\gamma, g):=\widehat{\Psi}_{-}(\gamma, g)[\delta(\gamma)]\left[\gamma_{+}\right] \in M^{(\infty)}(\delta(\gamma)) \\
& \Psi_{+}(\gamma, g):=\widehat{\Psi}_{+}(\gamma, g)[\delta(\gamma)]\left[\gamma_{-}\right] \in M^{(0)}(\delta(\gamma))
\end{aligned}
$$

и пара $\left(\Psi_{+}, \Psi_{-}\right)$является парой волновых матриц типа $[\delta(\gamma)]$ для двумерной иерархии Тоды. Дифференцируя левую часть уравнения (20) по $t_{i \alpha}$, получаем

$$
\begin{aligned}
& \left\{\partial_{i \alpha} \widehat{\Psi}_{-}(\gamma, g)+\widehat{\Psi}_{-}(\gamma, g) \Lambda^{i k} i_{k}\left(E_{\alpha}\right)\right\}\left[\gamma_{+}\right][g]= \\
& \quad=\left\{\partial_{i \alpha} \widehat{\Psi}_{-}(\gamma, g) \cdot \widehat{\Psi}_{-}(\gamma, g)^{-1}+\mathcal{L}\left(\widehat{\Psi}_{-}\right)^{i} U_{\alpha}\left(\widehat{\Psi}_{-}\right)\right\} \Psi_{-}(\gamma, g)[g]
\end{aligned}
$$

Применяя $\partial_{i \alpha}$ к правой части, получаем

$$
\partial_{i \alpha} \widehat{\Psi}_{+}(\gamma, g) \cdot[\delta(\gamma)]\left[\gamma_{-}\right]=\left\{\partial_{i \alpha} \widehat{\Psi}_{+}(\gamma, g) \cdot \widehat{\Psi}_{+}(\gamma, g)^{-1}\right\} \Psi_{+}(\gamma, g),
$$

и комбинация обоих выражений дает

$$
\partial_{i \alpha} \widehat{\Psi}_{-}(\gamma, g) \cdot \widehat{\Psi}_{-}(\gamma, g)^{-1}+\mathcal{L}\left(\widehat{\Psi}_{-}\right)^{i} U_{\alpha}\left(\widehat{\Psi}_{-}\right)=\partial_{i \alpha} \widehat{\Psi}_{+}(\gamma, g) \cdot \widehat{\Psi}_{+}(\gamma, g)^{-1}
$$


Теперь старший коэффициент матрицы $\widehat{\Psi}_{-}(\gamma, g) \in L T_{0}(R)$ равен единице, откуда следует, что $\partial_{i \alpha} \widehat{\Psi}_{-}(\gamma, g) \cdot \widehat{\Psi}_{-}(\gamma, g)^{-1} \in L T_{-1}(R)$. Тогда из уравнения (21) мы заключаем, что выполняются соотношения

$$
\begin{aligned}
& \left(\mathcal{L}\left(\widehat{\Psi}_{-}\right)^{i} U_{\alpha}\left(\widehat{\Psi}_{-}\right)\right)_{+}=\partial_{i \alpha} \widehat{\Psi}_{+}(\gamma, g) \cdot \widehat{\Psi}_{+}(\gamma, g)^{-1} \\
& \partial_{i \alpha} \widehat{\Psi}_{-}(\gamma, g) \cdot \widehat{\Psi}_{-}(\gamma, g)^{-1}=\left(\mathcal{L}\left(\widehat{\Psi}_{-}\right)^{i} U_{\alpha}\left(\widehat{\Psi}_{-}\right)\right)_{-} .
\end{aligned}
$$

В частности, отсюда следует, что часть уравнений линеаризации удовлетворяется для $\Psi_{+}$и $\Psi_{-}$:

$$
\partial_{i \alpha} \Psi_{+}=\left(\mathcal{L}\left(\widehat{\Psi}_{-}\right)^{i} U_{\alpha}\left(\widehat{\Psi}_{-}\right)\right)_{+}\left(\Psi_{+}\right), \quad \partial_{i \alpha} \Psi_{-}=\left(\mathcal{L}\left(\widehat{\Psi}_{-}\right)^{i} U_{\alpha}\left(\widehat{\Psi}_{-}\right)\right)_{+}\left(\Psi_{-}\right) .
$$

Для параметров $s_{j \beta}$ мы действуем аналогичным образом: дифференцируем обе части уравнения (20), в результате получаем

$$
\begin{aligned}
& \left(\mathcal{M}\left(\widehat{\Psi}_{+}\right)^{j} V_{\beta}\left(\widehat{\Psi}_{+}\right)\right)_{-}=\partial_{j \beta} \widehat{\Psi}_{-}(\gamma, g) \cdot \widehat{\Psi}_{-}(\gamma, g)^{-1} \\
& \left(\mathcal{M}\left(\widehat{\Psi}_{+}\right)^{j} V_{\beta}\left(\widehat{\Psi}_{+}\right)\right)_{+}=\partial_{j \beta} \widehat{\Psi}_{+}(\gamma, g) \cdot \widehat{\Psi}_{+}(\gamma, g)^{-1}
\end{aligned}
$$

откуда следуют оставшиеся уравнения для $\Psi_{+}$и $\Psi_{-}$:

$$
\partial_{j \beta} \Psi_{+}=\left(\mathcal{M}\left(\widehat{\Psi}_{+}\right)^{j} V_{\beta}\left(\widehat{\Psi}_{+}\right)\right)_{-}\left(\Psi_{+}\right), \quad \partial_{j \beta} \Psi_{-}=\left(\mathcal{M}\left(\widehat{\Psi}_{+}\right)^{i} V_{\beta}\left(\widehat{\Psi}_{+}\right)\right)_{-}\left(\Psi_{-}\right) .
$$

Поскольку $\widehat{\Psi}_{-}(\gamma, g):=\Phi_{-}(\gamma, g)\left[\gamma_{-}\right]$и $\widehat{\Psi}_{+}(\gamma, g):=\Phi_{-}(\gamma, g)\left[\gamma_{+}\right]$, возмущения удовлетворяют соотношениям

$$
\mathcal{L}\left(\widehat{\Psi}_{-}\right)=\mathcal{L}\left(\Phi_{-}\right), \quad U_{\alpha}\left(\widehat{\Psi}_{-}\right)=U_{\alpha}\left(\Phi_{-}\right), \quad \mathcal{M}\left(\widehat{\Psi}_{+}\right)=\mathcal{M}\left(\Phi_{+}\right), \quad V_{\beta}\left(\widehat{\Psi}_{+}\right)=V_{\beta}\left(\Phi_{+}\right) .
$$

Окончательный результат теперь приобретает вид сформулированной во введении теоремы, в которой разложение $\left[\gamma g \gamma^{-1}\right]=\Phi_{-}^{-1} \Phi_{+}$соответствует утверждению 4.1.

Благодарности. Работа выполнена в рамках программы NWO-РФФИ "Геометрические аспекты квантовой теории и интегрируемых систем” (проект № 047.017.015).

\section{Список литературы}

[1] S. Kharchev, A. Marshakov, A. Mironov, A. Morozov, Nucl. Phys. B, 397:1-2 (1993), 339-378.

[2] K. Ueno, K. Takasaki, "Toda lattice hierarchy", Group Representations and Systems of Differential Equations (Tokyo, 1982), Adv. Stud. Pure Math., 4, ed. K. Okamoto, North-Holland, Amsterdam-New York, 1984, 1-95.

[3] G. F. Helminck, Acta Appl. Math., 90:1-2 (2006), 121-142.

[4] G. F. Helminck, M. G. Mishina, S. V. Polenkova, Bulg. J. Phys., 33:Suppl. 2 (2006), 600-611.

[5] M. Mulase, J. Diff. Geom., 19:2 (1984), 403-430.

[6] G. Segal, G. Wilson, Publ. Math. IHES, 61 (1985), 5-65.

[7] G.F. Helminck, S. V. Polenkova, " $\tau$-Functions for the two-dimensional infinite Todahierarchy associated with a commutative algebra", in preparation.

[8] A. Grothendieck, Bull. Soc. Math. France, 84 (1956), 319-384. 